\title{
La recherche en éducation médicale : état des lieux, perspectives et rôle de la Société internationale francophone d'éducation médicale (SIFEM)
}

\author{
Medical education research: overview, outlook and role of the Société \\ internationale francophone d'éducation médicale (SIFEM) (French-speaking \\ medical education society)
}

\section{Thierry PELACCIA ${ }^{1,2}$, Valérie DORY ${ }^{3}$, et Jean-François DENEF $^{4}$}

1 Service d'aide médicale urgente (SAMU 67) et Centre d'enseignement des soins d'urgence (CESU 67) - Hôpitaux universitaires de Strasbourg, Strasbourg, France

2 Laboratoire de recherche en pédagogie des sciences de la santé, Département de pédagogie médicale - Faculté de médecine de Strasbourg, Strasbourg, France

3 Chargée de recherches du F.R.S.-FNRS, Institut de recherche santé et société, Université catholique de Louvain, Bruxelles, Belgique

4 Faculté de médecine et de dentisterie, Université catholique de Louvain, Bruxelles, Belgique

Manuscrit reçu le 28 juillet 2011 ; commentaires éditoriaux formulés aux auteurs le 12 et le 17 octobre 2011 ; accepté pour publication le 19 octobre 2011

Mots-clés
SIFEM ;
recherche
en éducation
médicale;
pédagogie médicale

Résumé - Contexte : Les pratiques de recherche en éducation médicale sont régulièrement mises en question dans le monde francophone, en raison de l'ancrage traditionnellement positiviste de la recherche bio-médicale qui influence considérablement les chercheurs issus de ce milieu, du déficit de formations spécificiquement dédiées à la recherche en éducation des sciences de la santé et d'un financement insuffisant et inadéquat. Ceci se traduit par une remarquable absence des chercheurs francophones dans le paysage international de la recherche en éducation médicale. But : Dans cet article, nous présentons et discutons plusieurs propositions validées par le groupe recherche de la Société internationale francophone d'éducation médicale (SIFEM), dans le but de développer et de promouvoir une recherche en éducation médicale de qualité dans le monde francophone. Méthode : Les membres du groupe recherche de la SIFEM ont été sollicités afin de donner leurs avis et d'exprimer leur degré d'accord par rapport à 12 propositions formulées dans un document accessible sur Internet. Résultats : Plusieurs propositions sont unanimement ou très majoritairement retenues par les répondants. Elles ont pour but d'intéresser les jeunes enseignants à la recherche en éducation médicale, d'augmenter la qualité des travaux et de valoriser la production scientifique du champ. Conclusion : Sur la base de ces propositions, la SIFEM souhaite s'engager dans des actions de terrain visant à promouvoir la pratique d'une recherche de qualité en éducation médicale. 


$\begin{array}{ll}\text { Keywords } & \text { Abstract - Background: Medical education research is regularly challenged, especially in } \\ \text { SIFEM; } & \text { French-speaking countries. Its limitations have been attributed to the traditionally positivist } \\ \text { research in medical } & \text { stance of its scientists, unsuited training as well as a lack of proper financing of its underta- } \\ \text { education; } & \text { kings. French-speaking scientists have been conspicuously absent from the medical educa- } \\ \text { medical education } & \text { tion scene. Aims: This paper presents and discusses proposals made by members of the } \\ & \text { SIFEM (international French-speaking medical education society) research task group in } \\ & \text { order to foster and promote quality medical education research in the French-speaking world. } \\ & \text { Methods: Members of the SIFEM research task group were asked what they thought of } \\ & \text { 12 proposals available online and how much they agreed with these statements. Results: } \\ & \text { Respondents unanimously or widely agreed with a number of proposals aimed at involving } \\ & \text { young teachers in medical education research, increasing the quality of research and status of } \\ & \text { field output. Conclusion: SIFEM plans to carry out field work to promote quality medical } \\ & \text { education research on the basis of these proposals. }\end{array}$

\section{Introduction}

La recherche en éducation médicale désigne toute démarche menée selon une méthodologie systématique et donnant lieu à la production de connaissances généralisables ou transférables. Albert distingue les travaux de recherche selon leur finalité principale qui peut être respectivement, soit d'améliorer les pratiques enseignantes des différents intervenants impliqués dans la formation des médecins, auquel cas il parle de production scientifique «pour les utilisateurs », soit de développer le corpus des savoirs savants disponibles pour les chercheurs en éducation médicale eux-mêmes, auquel cas il parle de production scientifique « pour les producteurs » ${ }^{[1,2]}$.

Deux clarifications terminologiques doivent d'emblée être mises en exergue concernant la locution "éducation médicale». La première concerne le terme «éducation», qu'il nous semble préférable d'utiliser, parce qu'il témoigne mieux du caractère pluridisciplinaire du champ et de la diversité des thématiques explorées par les chercheurs, plutôt que le terme «pédagogie », qui est pourtant plus couramment usité en contexte francophone mais qui est plus réducteur car il se réfère essentiellement aux interactions développées dans le cadre des activités d'enseignement et d'apprentissage. La seconde concerne le qualificatif qui est annexé à ce terme « médicale »-, qui doit être entendu au sens générique comme se rapportant à l'ensemble des professions de santé et pas exclusivement aux médecins, même si nous ciblerons essentiellement la discussion sur la formation des médecins, envisagée à titre prototypique.

Précisément, nous tenterons dans un premier temps d'identifier les objets de recherche de l'éducation médicale. Nous adopterons ensuite une posture critique quant aux pratiques des chercheurs du champ. Nous discuterons enfin du rôle de la Société internationale francophone d'éducation médicale (SIFEM) dans la promotion, la valorisation et la disciplinarisation de la recherche en éducation médicale en milieu francophone, ce néologisme de disciplinarisation étant employé pour désigner le processus, socialement et historiquement situé, de construction et de reconnaissance de ce champ en tant que discipline légitime au regard de sa spécificité et de son autonomie. Il convient cependant de noter que beaucoup d'autres professionnels de santé ont investi de façon remarquable le domaine de la recherche en éducation - en particulier en Amérique du Nord comme en atteste le dynamisme de revues telles que «The Journal of Nursing Education », «The Journal of Dental Education » ou encore «The Journal of Veterinary Medical Education ». 


\section{Les objectifs de la recherche en éducation médicale : " Mieux comprendre pour qu'ils apprennent mieux »!}

Les objectifs de la recherche en éducation médicale sont multiples et ses enjeux s'inscrivent à plusieurs niveaux :

- À l'échelle des acteurs de la formation médicale, il s'agit de sous-tendre scientifiquement les pratiques éducatives. Norman ${ }^{[3]}$ écrivait à ce sujet en 2002 qu' « avant les années soixante-dix, les avancées dans le domaine de l'éducation étaient essentiellement adoptées sur la base d'efforts de persuasion et de décisions politiques. Depuis cette époque, les changements sont plus souvent initiés ou déterminés sur la base de la preuve ». Cette évolution est en fait similaire à celle de la pratique médicale, qui a vu naître dans les années soixantedix la notion de «médecine fondée sur les preuves»( «Evidence-Based Medicine »), dans laquelle la démarche diagnostique et les choix thérapeutiques reposent sur les dernières données de la littérature scientifique, afin d'optimiser la prise en charge individuelle des patients ${ }^{[4]}$. Le concept d'éducation médicale fondée sur les preuves ( «Best evidence medical education ») a quant à lui fait son apparition à l'aube des années deuxmille ${ }^{[5]}$. Il désigne une démarche devenue essentielle dans un environnement éducatif marqué par une préoccupation d'efficience et par des attentes fortes de la part de la société. La recherche en éducation médicale veut remettre en cause le principe historique d'une approche empirique et intuitive de l'enseignement. Elle a pour but de valoriser une attitude scientifique basée sur des travaux de recherche rigoureux qui permettent en quelque sorte de «rationaliser» les pratiques éducatives. $\mathrm{Au}$ même titre que le réputé institut de recherche bruxellois De Duve a pour devise «Mieux comprendre pour mieux soigner $»$, la recherche en éducation médicale se doit de mieux comprendre les processus d'apprentissage pour rendre l'enseignement plus efficace. Il s'agit de « Mieux comprendre pour mieux enseigner» ou, plus encore, de «Mieux comprendre pour qu'ils apprennent mieux !».

- À l'échelle institutionnelle, la pratique et la promotion de la recherche en éducation médicale permettent aux facultés de médecine d'acquérir un «leadership » et une reconnaissance de la part des autres institutions et de la communauté scientifique du domaine. Comme le souligne à ce sujet Charlin, si l'innovation pédagogique est une démarche intrinsèquement valorisante pour les structures de formation, elle atteint rapidement ses limites dès lors qu'elle n'est pas associée à des efforts de recherche ${ }^{[6]}$.

- À l'échelle de la discipline, il s'agit d'accompagner le développement de l'éducation médicale ${ }^{[6]}$. L'innovation en éducation médicale ne peut en effet être envisagée indépendamment des activités de recherche et de la production de connaissances, qui sont une condition nécessaire à la valorisation académique de cette discipline ${ }^{[7]}$. Au regard du caractère insuffisamment développé de l'approche scientifique en éducation médicale, les démarches de recherche au sein du champ revêtent une importance cruciale ${ }^{[7]}$.

- À l'échelle sociétale, l'objectif est d'améliorer, à travers des démarches éducatives plus efficaces et plus pertinentes, les pratiques de soins et, in fine, la santé des populations.

\section{Quels sont les objets de recherche de l'éducation medicale?}

Il convient d'emblée de souligner les difficultés à identifier de manière consensuelle les objets de recherche en éducation médicale ${ }^{[7]}$. Cette situation peut probablement être attribuée au caractère fondamentalement et historiquement pluridisciplinaire du champ, qui voit coexister - et, parfois, collaborer des professionnels de santé, des spécialistes de l'éducation, des psychologues, des sociologues et de nombreux autres acteurs issus des sciences humaines et sociales. Chaque discipline a évidemment apporté ses propres objets de recherche et il est aisément 
imaginable que les préoccupations des enseignantscliniciens sont naturellement éloignées de celles des anthropologues. Le caractère éclectique des ancrages disciplinaires des chercheurs en éducation médicale est à l'origine de tensions et d'affrontements associés à des enjeux de pouvoir. Dans un paysage de la recherche en éducation médicale d'apparence parfois chaotique, l'identification des objets d'étude se révèle hautement complexe et incertaine. Nous ne ferons ainsi qu'esquisser les lignes directrices qui structurent le champ.

Les préoccupations des chercheurs en éducation médicale sont avant tout d'ordre pédagogique, ce qui explique que dans le monde francophone, le champ soit souvent désigné sous le terme de «pédagogie médicale ». Il s'agit ainsi de s'intéresser prioritairement aux modalités de planification pédagogique, aux activités d'enseignement et d'apprentissage et aux pratiques d'évaluation des apprentissages et des dispositifs de formation au sein des facultés de médecine ${ }^{[8-10]}$. Le caractère hétéronome de la régulation des pratiques de recherche du champ constitue une explication probable à cette situation. Ces pratiques sont en effet largement influencées par les facultés de médecine et par leurs préoccupations évidemment légitimes - quant à la qualité de la formation. Cette dépendance est à la fois historique dans la mesure où la recherche en éducation médicale s'est développée sur la base de pratiques éducatives qui ont précédé la naissance du champ - et économique, puisque le financement de ce dernier repose essentiellement sur des fonds universitaires ${ }^{[7,11]}$.

L'éducation médicale ouvre toutefois la voie à des travaux de recherche dont les objets sont beaucoup plus vastes que ceux de la pédagogie médicale, notamment grâce au caractère pluridisciplinaire du champ. La prise de décision et l'expertise, les déterminants psychosociaux de la formation médicale, la construction identitaire des étudiants en sciences de la santé, la collaboration et la communication interprofessionnelles, la professionnalisation, ou encore le leadership et le management institutionnels, constituent autant d'objets relevant légitimement des mandats de recherche du champ. Certains sont d'ailleurs explorés depuis plusieurs dizaines d'années.

\section{Quelles sont les pratiques des acteurs du champ de la recherche en éduca- tion médicale?}

L'éducation médicale devenant progressivement une discipline à part entière, le champ de sa recherche et les pratiques des chercheurs qui l'investissent font fréquemment l'objet de critiques que nous souhaiterions discuter et qui ont pour certaines déjà été soulignées dans une précédente publication ${ }^{[7]}$. Ces critiques sont notamment liées au rôle central des médecins dans la structuration et le développement du champ, qui a naturellement conduit chercheurs et enseignantscliniciens à transposer des pratiques de recherche clinique ou fondamentale aux pratiques de recherche en éducation, alors qu'elles se révèlent en partie inappropriées au regard des objets de recherche de l'éducation médicale.

La recherche en éducation médicale est le plus souventet implicitement pratiquée selon une tradition positiviste et a-théorique de la recherche scientifique

Toute démarche de recherche est en principe adossée à une posture théorique - ou paradigmatique - qui peut se définir selon les réponses apportées à trois questionnements :

- le questionnement ontologique, qui renvoie à la nature de la réalité à connaître (par exemple, existe-t-elle indépendamment du regard que portent sur elle les individus ou est-elle construite par ces derniers ?)

- le questionnement épistémologique, qui interroge la façon dont les auteurs appréhendent la production de connaissances visant à rendre compte de cette réalité (par exemple en tentant de l'observer de façon neutre et à distance ou en s'autorisant des interactions avec elle)

- le questionnement méthodologique, relatif aux modalités de recueil et d'analyse des données.

À titre d'exemple, le tableau I distingue de façon didactique le positivisme et le constructivisme du point de vue des réponses apportées respectivement aux questionnements ontologique, épistémologique et méthodologique. 
Tableau I. Comparaison du positivisme et du constructivisme d'un point de vue du point de vue des questionnements ontologique, épistémologique et méthodologique.

\begin{tabular}{lcc}
\hline & Positivisme & Constructivisme \\
\hline $\begin{array}{l}\text { Òtologie (nature de la réalité } \\
\text { ànnaitre) }\end{array}$ & $\begin{array}{c}\text { La réalité existe indépendamment du } \\
\text { sujet (réaliste) }\end{array}$ & $\begin{array}{c}\text { Les réalités sont multiples et font l'objet } \\
\text { d'une construction spécifique et locale } \\
\text { (relativiste) }\end{array}$ \\
\hline $\begin{array}{l}\text { Épistémologie (nature de la } \\
\text { connaissance à produire au }\end{array}$ & $\begin{array}{c}\text { La connaissance est extérieure à } \\
\text { l'individu et ce qui est observé est vrai la réalité à connaître) } \\
\text { (dualiste, objectiviste) }\end{array}$ & $\begin{array}{c}\text { La connaissance est le fruit d'une } \\
\text { (co)construction par l'individu et ce qui } \\
\text { est observé est créé ([inter]subjectiviste) }\end{array}$ \\
\hline $\begin{array}{l}\text { Méthodologie (moyens à } \\
\text { mettre en œuvre pour produire } \\
\text { la connaissance) }\end{array}$ & $\begin{array}{c}\text { Quantitative expérimentale } \\
\text { (vérification d'hypothèses considérées } \\
\text { comme des faits ou des lois) }\end{array}$ & \begin{tabular}{c} 
Qualitative et quantitative \\
\hline
\end{tabular}
\end{tabular}

Le choix et le caractère explicite de ces postures constituent les fondements d'une approche holistique, réfléchie et cohérente de l'objet de recherche ${ }^{[12]}$. Elle est en quelque sorte « l'angle de vue » adopté par le chercheur. Comme le souligne à ce sujet Ringsted, « ce qui fait d'une étude un travail de recherche est son inscription dans un cadre conceptuel ou théorique de l'apprentissage et de l'éducation, et le choix d'une approche de recherche $[. .].{ }{ }^{[13]}$.

Le dogme positiviste et son corollaire méthodologique quantitatif dominent incontestablement le champ de la recherche en éducation médicale, en raison de sa prééminence dans le domaine de la recherche clinique et fondamentale ${ }^{[14]}$. Or, il atteint évidemment rapidement ses limites lorsqu'il s'agit d'appréhender la complexité du « fait » éducatif. La pertinence de cette posture monolithique a ainsi largement été remise en cause depuis les années quatrevingt ${ }^{[15]}$. Si le paradigme constructiviste tend depuis à occuper une place croissante, il n'en demeure pas moins secondaire et, parfois, critiqué par certains acteurs influents de la recherche en éducation médicale, comme le soulignent Pelaccia et Paillé ${ }^{[16]}$. Un nombre croissant de chercheurs du champ plaide toutefois aujourd'hui en faveur de la doctrine du "bestfit", qui consiste à choisir le paradigme et la méthode les plus appropriés pour répondre à la question de recherche.

Par ailleurs, les chercheurs du champ ont fréquemment tendance à sous-estimer l'importance du choix d'une posture théorique ${ }^{[17-20]}$ et, par conséquent, à sous-dimensionner la place accordée à la conceptualisation de leurs travaux. La démarche de recherche en éducation implique la réalisation de plusieurs étapes incluant l'exploration de la littérature scientifique, l'élaboration de la problématique et la définition de la méthode. Viennent ensuite la collecte des données, leur analyse, la confrontation critique des résultats avec la théorie et, enfin, la diffusion et la valorisation du travail sous forme de communications ou de publications. Dans ce parcours, la théorisation constitue une étape essentielle et obligatoire de la problématisation. Elle guide également les chercheurs dans leurs choix méthodologiques et dans l'interprétation des résultats. Or, les chercheurs en éducation médicale restreignent fréquemment leur démarche à l'étape d'observation (il s'agit, par exemple, de décrire un nouveau dispositif pédagogique) et de vérification (il s'agit des études dites de « justification », qui visent, par exemple, à démontrer l'efficacité d'une approche pédagogique) ${ }^{[12-21]}$. L'absence de conceptualisation est préjudiciable à l'échelle de la discipline car elle limite l'émergence de véritables communautés scientifiques œuvrant autour d'objets de recherche communs et pouvant appuyer leur démarche sur des travaux antérieurs réalisés autour du même objet ${ }^{[10,21,22]}$.

\section{Les chercheurs en éducation médicale} sont insuffisamment formés

Les difficultés de certains chercheurs du champ à renoncer à une vision exclusivement positiviste ou postpositiviste du monde et de la connaissance, à élargir leur spectre méthodologique et à s'approprier 
des théories et des concepts souvent issus du champ des sciences humaines et sociales sont en grande partie expliquées par leur manque de formation.

En effet, les professionnels de santé qui investissent le champ de la recherche en éducation médicale sont fréquemment des cliniciens ou des chercheurs en sciences fondamentales. Ils ont rarement bénéficié d'une formation spécifique à la démarche de recherche dans ce champ. Les formations en éducation qui leur sont proposées ne revêtent ainsi dans certains pays pas de caractère obligatoire et ciblent le plus souvent les aspects pratiques et pédagogiques de l'enseignement et de l'apprentissage des sciences médicales. C'est notamment le cas des diplômes universitaires de pédagogie médicale, qui constituent en France le dispositif principal de formation complémentaire des médecins.

Or, la posture et la démarche de recherche en éducation médicale ne sont pas superposables à celles qui caractérisent la recherche clinique ou fondamentale. Elles s'en singularisent par plusieurs aspects - en partie exposés dans la précédente section - ce qui implique la mobilisation de compétences spécifiques, idéalement acquises dans le cadre d'une formation académique.

La recherche en éducation médicale est pratiquée de façon dispersée, avec des ressources financières limitées

Une majorité de travaux de recherche en éducation médicale est réalisée sans ressources financières ou avec des ressources internes provenant des institutions qui emploient les chercheurs ${ }^{[2,8,9]}$. Or, l'augmentation des fonds attribués aux travaux de recherche en éducation médicale est considérée comme une stratégie fondamentale d'amélioration de la qualité de la production scientifique du champ ${ }^{[23]}$. Par ailleurs, le mode de financement essentiellement interne conduit de toute évidence les chercheurs à ancrer leurs démarches dans un contexte local qui limite fortement la portée de leurs études.

Il est également intéressant de souligner que moins du tiers des auteurs publiant des travaux dans ce champ revendique une appartenance à un département de pédagogie médicale ${ }^{[23]}$. Pourtant, la collaboration des cliniciens-chercheurs avec ces structures permettrait de conduire leurs études de façon plus rigoureuse, au même titre que la création de centres nationaux d'excellence dans le domaine de la recherche en éducation, qui faciliteraient et dynamiseraient en outre les liens entre les acteurs du champ ${ }^{[23]}$. Il est à ce sujet important de souligner la pertinence, dans la démarche de recherche en éducation médicale, de la collaboration entre les experts de « contexte » et de «contenu » (les enseignants des facultés de médecine) et les experts de "processus » (les chercheurs en éducation).

Le monde francophone est remarquablement absent du paysage de la recherche en éducation médicale

Charlin soulignait en 2007 que « nous [les chercheurs francophones] sommes remarquablement absents de la scène internationale en matière d'éducation appliquée aux sciences de la santé » ${ }^{[6]}$. Une grande majorité des centres de recherche dans ce champ se concentre en effet au Canada, aux États-Unis, aux Pays-Bas et en Grande-Bretagne. Le monde francophone - en dehors de l'Amérique du Nord - est ainsi peu actif, tant sur le plan de la production, que de la publication scientifique. L'absence fréquente, au sein des facultés de médecine, de comités d'éthique qualifiés pour évaluer ce type de travaux constitue une difficulté supplémentaire pour les chercheurs francophones, au regard des exigences récemment renforcées des revues d'éducation médicale sur le plan du respect des règles dans ce domaine.

\section{Quelles sont les propositions de la SIFEM pour promouvoir la recherche en éducation médicale dans le monde francophone?}

\section{Le groupe recherche de la SIFEM}

L'état des lieux de la pratique de la recherche en éducation médicale a conduit la SIFEM à mobiliser un groupe de chercheurs, avec pour objectif principal 
Tableau II. Membres du groupe recherche de la SIFEM.

\begin{tabular}{lc}
\hline Nom & Provenance \\
\hline Mathieu Albert & Totonto (Canada) \\
\hline Catherine Bertrand & Créteil (France) \\
\hline Georges Bordage & Chicago (États-Unis) \\
\hline Martine Chamberland & Sherbrooke (Canada) \\
\hline Bernard Charlin & Montréal (Canada) \\
\hline Jean-François Denef & Bruxelles, UCL (Belgique) \\
\hline Valérie Dory & Bruxelles, UCL (Belgique) \\
\hline Anne Demeester & Marseille (France) \\
\hline Brian Hodges & Toronto (Canada) \\
\hline Mathieu Nendaz & Genève (Suisse) \\
\hline Thierry Pelaccia & Strasbourg (France) \\
\hline Pierre Pottier & Nantes (France) \\
\hline Christophe Segouin & Paris (France) \\
\hline
\end{tabular}

d'accompagner le développement du champ dans le monde francophone, en ciblant notamment les jeunes enseignants-cliniciens et chercheurs en sciences fondamentales.

Il s'agit plus précisément :

- d'intéresser ces acteurs à la recherche en éducation médicale.

- d'améliorer la qualité de la production scientifique du champ.

- de valoriser les travaux de recherche réalisés dans le monde francophone.

Le groupe recherche de la SIFEM est constitué d'experts internationaux de l'éducation médicale et de jeunes médecins, doctorants ou titulaires d'un doctorat en éducation (tableau II).

Les membres du groupe ont été sollicités afin de donner leur avis sur des solutions concrètes permettant d'aller au-delà du constat de difficultés que connait le champ de la recherche en éducation médicale dans le monde francophone.

\section{Méthode}

Une liste de propositions a été soumise aux 13 membres du groupe entre octobre et décembre 2010, par l'intermédiaire d'une plateforme
Internet dédiée. Les invitations à répondre ont été envoyées collectivement par courriel. Une relance a été adressée nominativement aux non-répondants. Le taux de participation est de 92,3\%. Le sondage comportait 12 propositions - rédigées par les trois auteurs de cet article - réparties en trois catégories correspondant aux objectifs cités dans la section précédente. Les chercheurs devaient exprimer leur degré d'accord sur une échelle de Likert graduée de 1 (pas du tout d'accord) à 5 (tout à fait d'accord). Ils pouvaient également associer à chaque catégorie de propositions et à chaque proposition des commentaires libres.

\section{Résultats}

Les résultats sont présentés dans le tableau III.

\section{Discussion}

Les membres du groupe recherche de la SIFEM ont été invités à s'exprimer sur trois axes de développement et de promotion de la recherche en éducation médicale dans le monde francophone.

\section{Axe 1 : intéresser un plus grand nombre de jeunes enseignants à la recherche en éducation médicale}

Afin d'intéresser un plus grand nombre d'enseignants des facultés de médecine à ce champ de recherche, les répondants recommandent majoritairement :

- d'offrir un soutien financier aux jeunes enseignants désirant mener des travaux de qualité, à travers l'attribution de bourses.

- de proposer aux facultés de médecine des séances d'informations relatives à la pratique de la recherche en éducation médicale.

- d'encourager dans ces institutions la création de journées de pédagogie médicale.

Concernant la dernière proposition, certains répondants soulignent l'importance du caractère pluridisciplinaire de ces journées, qui pourraient être organisées dans le cadre de dispositifs impliquant plusieurs institutions. Elles permettraient de diffuser les 
Tableau III. Réponses du groupe recherche de la SIFEM aux propositions formulées en ligne (92,3 \% de répondants).

$\begin{aligned} \text { Échelle de Likert } & \text { Lello }\end{aligned}$

$\begin{array}{llllll}1 & 2 & 3 & 4 & 5 & \mathbf{4 + 5}\end{array}$

\section{Propositions}

Comment intéresser un plus grand nombre de jeunes enseignants à la recherche en pédagogie médicale ?

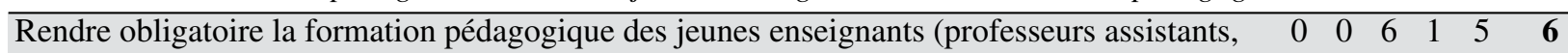
chefs de clinique, etc.), à travers des dispositifs diplômants (DIU, maîtrise, etc.)

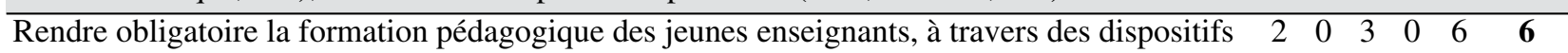
internes non diplômants au sein des facultés

Proposer des bourses aux jeunes enseignants souhaitant mettre en œuvre des projets de qualité $\quad \begin{array}{lllllllllll}0 & 0 & 0 & 0 & 12 & \mathbf{1 2}\end{array}$ dans le domaine de la recherche pédagogique

Créer des journées de pédagogie médicale dans les facultés des sciences de la santé, en y $\quad \begin{array}{llllllllll}0 & 1 & 0 & 1 & 10 & \mathbf{1 1}\end{array}$ invitant les jeunes enseignants

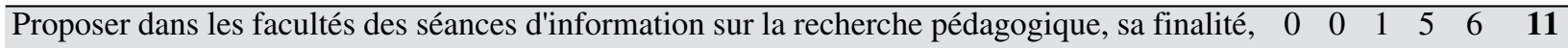
son intérêt, etc.

Comment améliorer la qualité de la recherche pédagogique pratiquée par les jeunes enseignants : pertinence du sujet, méthodologie, rédaction, etc.?

Proposer aux facultés des séminaires de recherche pédagogique «clés en main », qu'elles $\quad \begin{array}{llllllll}0 & 0 & 1 & 4 & 7 & \mathbf{1 1}\end{array}$ pourraient par exemple inclure dans les diplômes de pédagogie médicale

\begin{tabular}{lllllllllll}
\hline Organiser dans le cadre des congrès de pédagogie médicale des séminaires de recherche & & 0 & 0 & 0 & 4 & 8 & $\mathbf{1 2}$
\end{tabular}

Proposer, dans la revue Pédagogie Médicale, des articles et des fiches techniques permettant $\quad \begin{array}{lllllllllllll}0 & 0 & 1 & 10 & \mathbf{1 1}\end{array}$ de guider les enseignants dans l'élaboration, la réalisation et la valorisation d'un travail de recherche

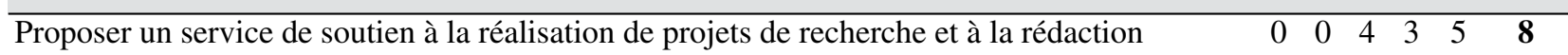
d'articles et de communications scientifiques

\begin{tabular}{|c|c|}
\hline Créer des projets de recherche multicentriques pilotés par la SIFEM & $\begin{array}{lllll}0 & 1 & 1 & 4 & 6\end{array}$ \\
\hline Comment améliorer la reconnaissance des travaux de recherche pédagogique réalisés $p$ & jeunes enseig \\
\hline $\begin{array}{l}\text { Inviter les jeunes enseignants à communiquer leurs résultats de recherche pédagogique à } \\
\text { l'occasion de journées de pédagogie médicale organisées dans les facultés }\end{array}$ & $\begin{array}{lllll}0 & 0 & 2 & 4 & 6\end{array}$ \\
\hline $\begin{array}{l}\text { Prendre en compte les travaux de recherche pédagogique dans le cadre de la nomination } \\
\text { la promotion des jeunes enseignants }\end{array}$ & $\begin{array}{llllll}0 & 0 & 1 & 0 & 11 & \mathbf{1 1}\end{array}$ \\
\hline
\end{tabular}

résultats des travaux qui y sont menés et de créer des opportunités de collaborations interdisciplinaires, interprofessionnelles et interinstitutionnelles, incluant les universités francophones des pays émergents.

Les trois propositions nous paraissent essentielles au regard du constat que nous avons précédemment dressé quant aux pratiques de recherche en éducation médicale. Leur mise en œuvre permettrait de contribuer à remédier à la méconnaissance du champ par les acteurs issus des sciences de la santé, à leurs lacunes sur le plan théorique et méthodologique et au caractère inadéquat et très insuffisant du financement des travaux du champ.
Si les membres du groupe considèrent que tout enseignant devrait bénéficier d'une formation de base en pédagogie, ils sont plus partagés quant à son caractère obligatoire. Ces réticences sont liées au fait que les dispositifs existants sont parfois trop contraignants au regard des tâches cliniques et d'enseignement des médecins et qu'ils sont essentiellement dédiés à la pratique de la pédagogie médicale et à l'innovation dans ce domaine, et non à la recherche. Les répondants expriment également leurs craintes quant au caractère fréquemment a-théorique des enseignements de pédagogie délivrés dans les facultés de médecine, qui encourageraient par conformisme l'adoption de 
pratiques de recherche fondées sur le paradigme biomédical, dont les limites ont été soulignées précédemment. Dans tous les cas, ces formations devraient conduire à l'attribution de crédits qui pourraient être valorisés dans le cadre de dispositifs de niveau supérieur (par exemple, les masters).

Axe 2: améliorer la qualité de la recherche en éducation pratiquée par les jeunes enseignants

Dans une perspective d'augmentation de la qualité de la production scientifique en éducation médicale, les membres du groupe recherche de la SIFEM valorisent le principe de la création de séminaires de formation, qui pourraient être proposés aux facultés de médecine, par exemple, dans le cadre des diplômes interuniversitaires. Ces séminaires pourraient également être inscrits de façon systématique au programme des manifestations scientifiques du champ, notamment celles organisées par la SIFEM. Ils cibleraient en particulier les questions de problématisation et de conceptualisation des travaux de recherche. Au même titre que les journées de pédagogie médicale, ces séminaires devraient être associés à l'attribution de crédits de formation.

Les répondants valident également très majoritairement le principe de la publication, dans la revue Pédagogie Médicale, d'articles et de fiches techniques en lien avec la pratique de la recherche en éducation médicale.

Si les membres du groupe considèrent que les dispositifs de tutorat devraient être encouragés, certains soulignent les obstacles probables de mise en œuvre d'une telle proposition, notamment en raison des difficultés à identifier des personnes ressources qui bénéficieraient d'un temps suffisant à consacrer à cette tâche.

Axe 3 : améliorer la reconnaissance des travaux de recherche pédagogique réalisés par les jeunes enseignants

Sur le plan de la valorisation des travaux de recherche en éducation médicale menés par les acteurs du champ, les répondants considèrent que la SIFEM devrait militer en faveur de la prise en compte, par les décideurs, de ces travaux dans la nomination et la promotion des enseignants.

\section{Conclusion}

Malgré les difficultés que connait le champ de la recherche en éducation médicale, celui-ci est incontestablement engagé à l'échelle mondiale dans une dynamique de professionnalisation, d'institutionnalisation et de valorisation de ses activités. La plupart des pays francophones d'Europe et d'Afrique demeurent toutefois globalement à l'écart de ce processus. La SIFEM s'engage, à travers son groupe recherche, dans une démarche de promotion et de développement d'une pratique de qualité de la recherche en éducation médicale dans le monde francophone.

\section{Votre avis nous intéresse!}

Le groupe recherche de la SIFEM est à l'écoute de vos rétroactions quant aux propositions présentées dans cet article. Vous pouvez nous faire part de vos commentaires, de vos attentes et de vos suggestions en écrivant à thierry.pelaccia@wanadoo.fr. Elles seront attentivement considérées et feront l'objet d'une discussion au sein du groupe, dans le but de retenir les propositions définitives qui donneront lieu, dans les prochains mois, à leur mise en œuvre pratique par la SIFEM.

\section{Contributions}

Les trois auteurs ont conjointement mené des discussions sur le contenu et la structuration de l'article. Thierry Pelaccia et Valérie Dory ont recueilli et analysé les données. Thierry Pelaccia a rédigé une première version qui a fait l'objet d'une révision par Valérie Dory et par Jean-François Denef. La version finale du manuscrit a été approuvée par les trois auteurs. 


\section{Références}

1. Albert M. Understanding the debate on medical education research: a sociological perspective. Acad Med 2004;79:948-54.

2. Albert M, Hodges B, Regehr G. La recherche en éducation médicale : entre le service et la science. Pédagogie Médicale 2006;7:73-81.

3. Norman GF. Research in medical education: three decades of progress. BMJ 2002;324:1560-2

4. Sackett DL, Rosenberg WM, Gray JA, Haynes RB, Richardson WS. Evidence based medicine: what it is and what it isn't. BMJ 1996;312:71-2.

5. Hart IR. Editorial: Best evidence medical education. Med Teach 1999;21:453-4.

6. Charlin B. La recherche en éducation médicale: pourquoi, comment ? Conférence présentée lors du congrès IPM 2007, Strasbourg, 2007. [On-line] Disponible sur : http://www.canal-u.tv/producteurs/ canal_u_medecine/dossier_programmes/ pedagogie_des_sciences_medicales/ colloque_et_evenement/ internet_et_pedagogie_medicale_strasbourg_2007/ ipm_2007_la_recherche_en_education_medicale_ pourquoi_comment

7. Pelaccia T, Triby E. La pédagogie médicale est-elle une discipline ? Pédagogie Médicale 2011;12:121-32.

8. Bordage G. La recherche en pédagogie médicale en Amérique du Nord : tour d'horizon et perspectives. Pédagogie Médicale 2000;1:9-12.

9. Dimitroff A, Davis W. Content analysis of research in undergraduate medical education. Acad Med 1996;71:60-67.

10. Regehr G. Trends in medical education research. Acad Med 2004;79:939-47.

11. Anderson MD, Kanter SL. Medical education in the United States and Canada, 2010. Acad Med 2010;84:2-18.

12. Rees CE, Monrouxe LV. Theory in medical education research: how do we get there? Med Educ 2010;44:334-9.
13. Ringsted C. Research in medical education. Notfall Rettungsmed 2009;2:57-60.

14. Bunniss S, Kelly D. Research paradigms in medical education research. Med Educ 2010;44:358-66.

15. Harris IB. Qualitative methods. In: Norman GR, van der Vleuten CP et Newble DI (eds.). International handbook of research in medical education. Boston: Kluwer Academic, 2002:45-93.

16. Pelaccia T, Paillé P. La recherche qualitative en pédagogie médicale : histoire, pratique et légitimité. Pédagogie Médicale 2011;12:179-92

17. Bligh J. Nothing is but what is not. Med Educ 2003;37:184-5.

18. Colliver JA. Educational theory and medical education practice: a cautionary note for medical school faculty. Acad Med 2002;77:1217-20.

19. Kuper A, Reeves S, Albert M, Hodges BD. Assessment: do we need to broaden our methodological horizons? Med Educ 2007;41:1121-3.

20. Prideaux D, Spencer J. On theory in medical education. Med Educ 2000;34:888-9.

21. Cook DA, Bordage G, Henk GS. Description, justification and clarification: a framework for classifying the purposes of research in medical education. Med Educ 2008;42:128-33.

22. Schuwirth L, Colliver J, Gruppen L, Kreiter C, Mennin $\mathrm{S}$, Onishi $\mathrm{H}$ et al. Research in assessment: consensus statement and recommendations from the Ottawa 2010 Conference. Med Teach 2011;33:224-33.

23. Baernstein A, Liss HK, Carney PA, Elmore JG. Trends in study methods used in undergraduate medical education research, 1969-2007. JAMA 2007;298:1038-45.

Correspondance et offprints : Thierry Pelaccia. SAMU 67-CESU 67, Pôle logistique des Hôpitaux universitaires de Strasbourg, 70 rue de l'Engelbreit, 67200 Strasbourg, France. Mailto: thierry.pelaccia@wanadoo.fr 